\title{
Conference Poster
}

\section{An Experimental and Numerical Investigation of Drag Reduction Through Biomimetic Modelling}

Manz, A., Monir, S. and Jones, M.

This is a paper presented at the 7th IEEE Int. Conference on Internet Technologies and Applications ITA-17, Wrexham, UK, 12-15 September 2017.

Copyright of the author(s). Reproduced here with their permission and the permission of the conference organisers.

\section{Recommended citation:}

Manz, A., Monir, S. and Jones, M. (2017) 'An Experimental and Numerical Investigation of Drag Reduction Through Biomimetic Modelling'. In: Proc. 7th IEEE Int. Conference on Internet Technologies and Applications ITA17, Wrexham, UK, 12-15 September 2017, pp. 199-203. doi: 10.1109/ITECHA.2017.8101938 


\title{
An Experimental and Numerical Investigation of Drag Reduction Through Biomimetic Modelling
}

\author{
Andreas Manz, Shafiul Monir, Martyn Jones \\ School of Applied Science, Computing and Engineering, \\ Glyndwr University, Plas Coch, Mold Road, \\ Wrexham, LL11 2AW, UK
}

\begin{abstract}
This paper characterises flow around a circular cylinder and investigates the potential of a non-smooth surface to reduce air resistance in a Reynolds number range between $\operatorname{Re}_{d}=8.09 \cdot 10^{4}$ and $\operatorname{Re}_{d}=2.02 \cdot 10^{5}$. The two- and threedimensional numerical simulations were performed using a steady-state solution and the Reynolds-averaged Navier-Stokes (RANS) approaches. Three different mesh designs and four turbulence models with various treatments were assessed and compared against experimental data. A total of 26 uneven preparations in the two-dimensional analysis and two riblet structures in the three-dimensional analysis were designed to investigate the effect of drag reduction. The results reported hold the potential of uneven structures to reduce the air resistance in the case of a circular cylinder. This research further demonstrates that engineering applications can benefit from mimicking nature's details and functions.
\end{abstract}

Keywords—Non-smooth surface, bionic, drag reduction, CFD

\section{INTRODUCTION}

The rising price of fossil fuels, economic restrictions and advanced engineering applications are the major reasons why engineers and scientists are more interested in methods to improve the aerodynamic behaviour of moving objects. Passive flow methods like a change in the surface structure are considered to be an influencing aspect of the aerodynamic behaviour.

Living organisms were able to invent almost perfect structures [1] shaped by their ongoing interaction with nature [2]. Surfaces which exhibit advanced structures and materials, as well as sophisticated chemical properties, have evolved [2]. Zhao et al. [3] and Chen et al. [4] investigated the bioreplication mimicking shark skin and drag reduction ability in water (drag reduction of $12 \%$ to $18.6 \%$ ) while Dou et al. [5] analysed the drag reduction using irregularly distributed microscale caves imitated from fish scales (drag reduction of $10 \%$ ). Bechert and Hage [6] explored the flow direction aligned riblets mimicking shark skin about the influence of stiffness as well as the alignment of ribs and determined the most useful characteristic with aligned scales and a hard stiffness. Bechert et al. [7] focused on clarifying the optimal proportion of the height to spacing between streamwise aligned riblets. Conscientious experiments have determined that the optimal height is half the distance between the ribs $(h=0.50 \cdot s)[7]$.
Furthermore, various flow direction aligned uneven structures were analysed by Bechert et al. [7] (blade-shaped riblets and riblets with triangular cross-section), El-Samni et al. [8] (blade-shaped riblets), $\mathrm{Xu}$ et al. [9] (riblets with triangular cross-section), Martin and Bhushan [10] (continuous blade, sawtooth and scalloped riblets) and Tian et al. [11] (riblets, convex dome and dimple concave shapes on a body of revolution). A drag reduction up to $11.6 \%$ was obtained. Dean and Bhushan [12] and Bixler and Bhushan [13] experimentally investigated the internal flow behaviour using streamwise aligned uneven structures in a rectangular duct. Bogdanovic-Jovanovic [14] experimentally and numerically investigated in the aerodynamic influence of golf ball dimples on a sphere in various flow regimes while Chowdhury et al. [15] experimentally investigated the drag reduction using different dimple sizes on a sphere.

Zhang et al. [16] numerically studied flow past a circular cylinder with uneven structures in the form of polygonal and ridged modification around the circumference, as well as designs with wavy and o-ringed geometries, for a Reynolds number of $\mathrm{Re}=5000$. It was observed that circumferential preparations failed to reduce drag due to a low Reynolds number.

Cui and Fu [17] numerically studied crosswise to the flow direction aligned bionic structures with the shape of placoidshaped grooves inspired from shark skin, V-shaped grooves mimicked from the wing of jaegers, riblet-shaped grooves adapted from the skin of seals and ridge-shaped grooves like on the outside surface of shells. Simulations were performed with water as fluid and all models achieved a drag reduction.

With the development of increasing computer power, CFD is becoming a preferred tool used to design, analyse and optimise fluid interactions. The direct numerical simulation (DNS) and the large eddy simulation (LES) are the most accurate approaches for turbulent flows [18]. However, the Reynolds-averaged Navier-Stokes (RANS) approach is the most used solving model for practical engineering problems due to the limited requirements in computational power [18].

The purpose of this investigation has been to characterise the flow around a circular cylinder and to compare a smooth surface against a non-smooth surface with uneven preparations to assess a drag reducing potential. 


\section{NUMERICAL SETUP}

Numerical simulations were carried out using the software code ANSYS Workbench R17.0 Academic. The design of the numerical domain was developed according to spatial conditions from the wind tunnel experiment with the length of $1000 \mathrm{~mm}$ and height as well as length of $450 \mathrm{~mm}$ respectively.

Simulations were performed using a pressure-based solver. Operation conditions were specified according to the wind tunnel experiment. Furthermore, no slip shear condition was selected for walls. Pressure-velocity coupling was defined performing the SIMPLE scheme. Spatial discretisation was specified with pressure as second order, momentum as second order upwind and turbulent kinetic energy as well as turbulent dissipation rate as first order upwind. All numerical simulations were conducted with convergence absolute criteria where the equations residual values are lower than $10^{-3}$.

\section{A. Two-dimensional Modelling}

Three meshing designs (Fig. 2) were investigated for determining the most accurate approach: structured multiblock (quadrilateral elements), unstructured (triangular elements and inflation layer) and hybrid (quadrilateral and triangular elements).

Four different turbulence models with various treatments were assessed with respect to validate simulation values against experimental data: k-epsilon and k-omega, Transitional k-kl-omega and Transition SST. A total of 87 simulations were compared.

In order to analyse flow over a non-smooth surface, 26 two -dimensional designs were created. Uneven structures with triangular and semi-circular geometries in elevating and hollowing shapes were designed. In addition, the number of evenly distributed elements around the circular body $(n=80$, $n=160$ and $n=240$ ) and the size of the geometries ( $h=0.5 \mathrm{~mm}$ and $h=0.25 \mathrm{~mm}$ ) varied in the different designs. In addition, the characteristic geometry of a golf ball dimple, as detailed in Bogdanovic-Javonovic et al. [14] was applied in a further uneven design.

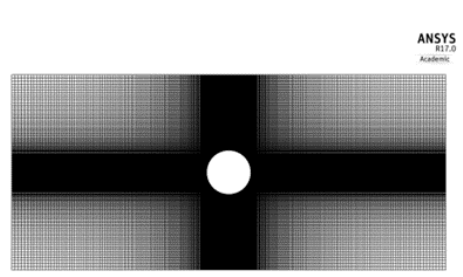

(a)

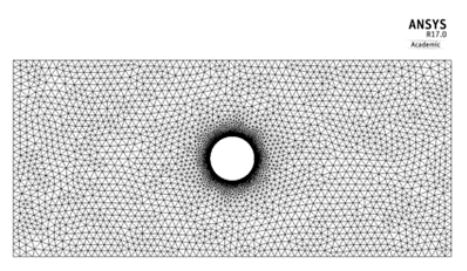

(c)

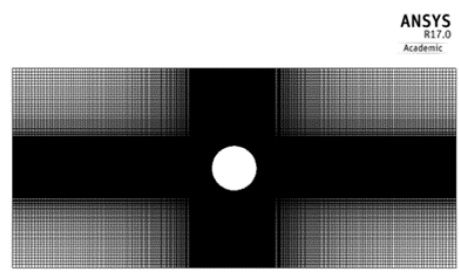

(e)

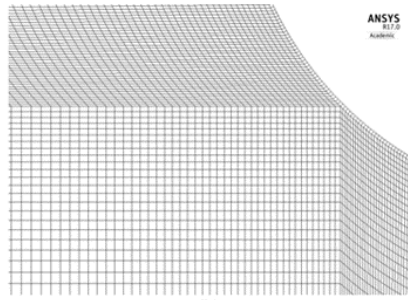

(b)

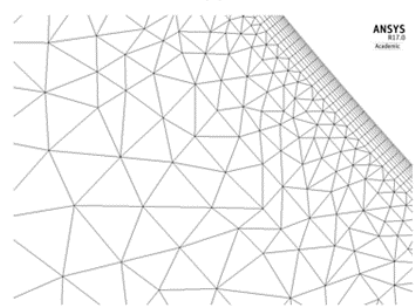

(d)

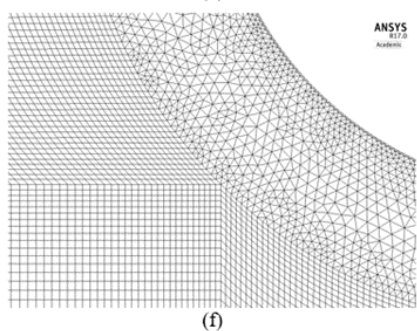

(f)
Fig. 2. The two-dimensional meshes generated: (a) structured multiblock mesh design, (b) close-up view of structured multiblock mesh, (c) unstructured mesh design, (d) close-up view of unstructured mesh, (e) hybrid mesh design, (f) close-up view of hybrid mesh.

\section{A. Three-dimensional Modelling}

An unstructured design with pure tetrahedral elements was defined. The generated mesh for the smooth circular cylinder did not exceed $450 \cdot 10^{3}$ mesh elements while in the case of the riblet structures the number of mesh elements was just below $10 \cdot 10^{6}$. The k-epsilon model with enhanced wall treatments was defined for all three-dimensional simulations.

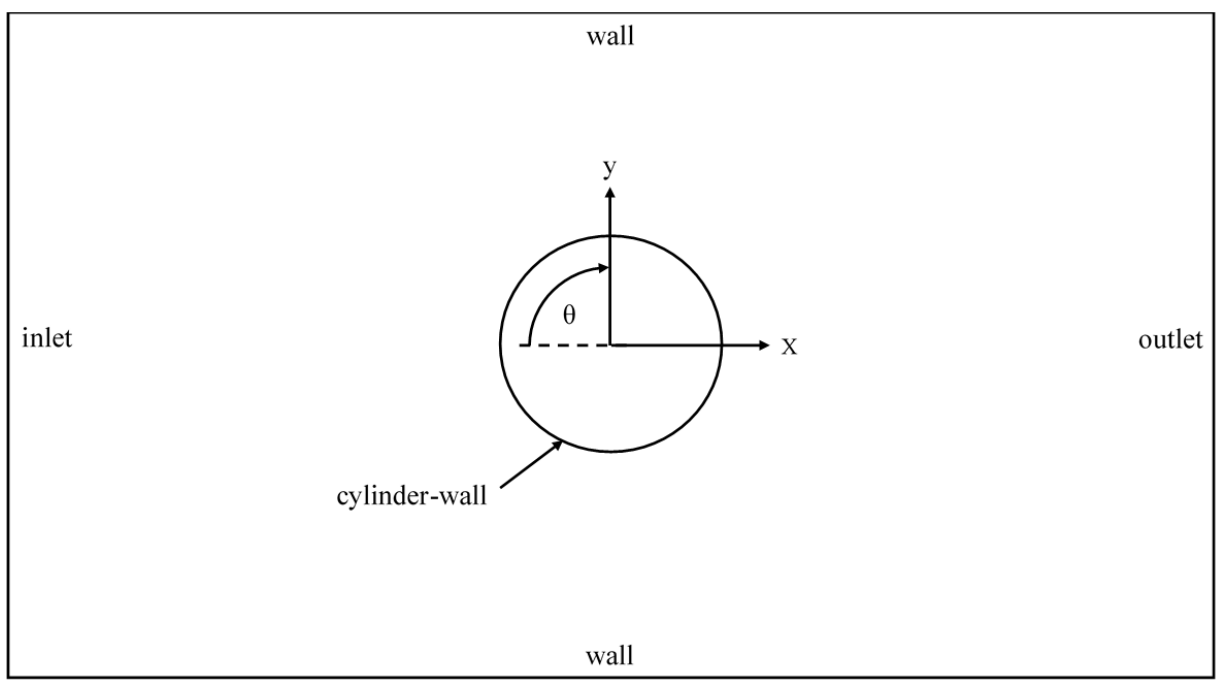

Fig. 1. Schematic of the two-dimensional numerical domain. 

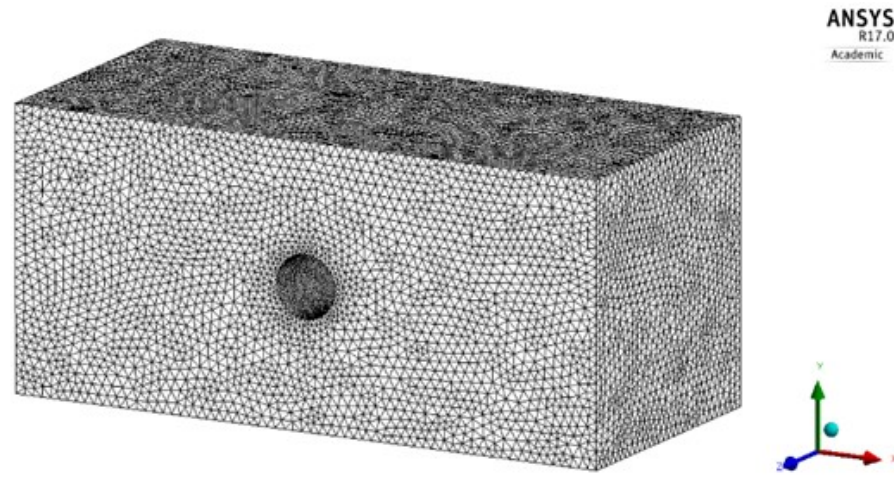

Fig. 3. Mesh generated of the three-dimensional numerical domain .

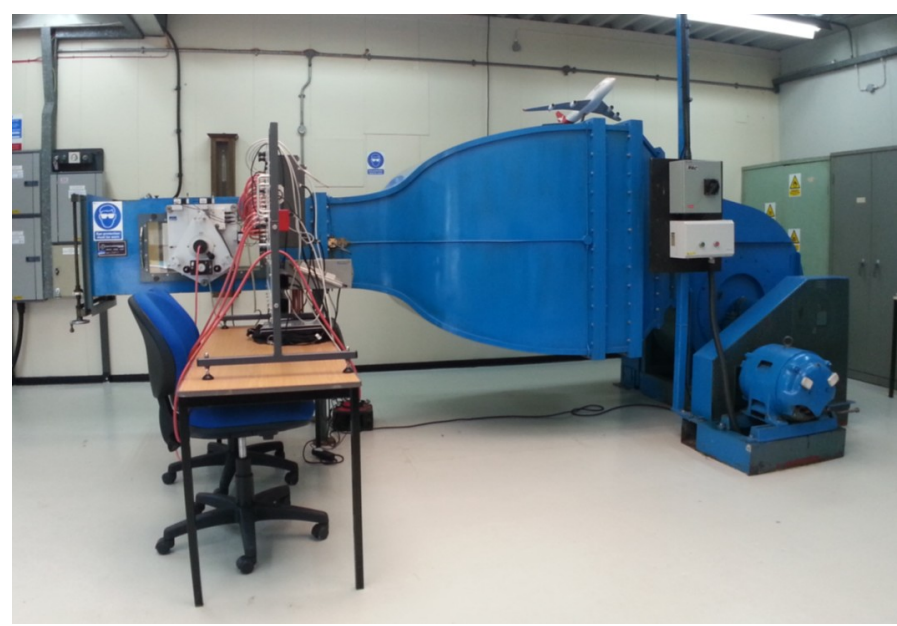

Fig. 4. Subsonic wind tunnel for the experimental research.

Two uneven structures were designed for the threedimensional analysis of air resistance reduction using streamwise aligned riblets. The design of the threedimensional geometry was defined as elevating equilateral triangles with an interior angle of 30 degrees. Furthermore, the relation of $h=0.5 \cdot \mathrm{s}$ suggested by Bechert et al. [7] was considered.

\section{EXPERIMENTAL SETUP}

A subsonic wind tunnel at Wrexham Glyndwr University was used for the experimental research (Fig. 4). Each setup was examined three times and average values were formed to reduce inaccuracy. One measurement of pressure coefficient was taken every 10 degrees of rotational angle. Ambient conditions were frequently measured during the experiment.

The pressure distribution around a smooth cylindrical body was conducted in five flow cases resulting in a Reynolds

Table I. Details of the ThreE-Dimensional Riblet Designs.

\begin{tabular}{|l|l|l|l|}
\hline \multicolumn{1}{|c|}{ Design } & \multicolumn{1}{|c|}{$\begin{array}{c}\text { Height }(\boldsymbol{h}) \\
{[\mathbf{m m}]}\end{array}$} & \multicolumn{1}{c|}{$\begin{array}{c}\text { Interior Angle } \\
\text { [degrees] }\end{array}$} & $\begin{array}{c}\text { Lateral Space (s) } \\
{[\mathbf{m m}]}\end{array}$ \\
\hline Riblet A & 0.5 & 30 & 1 \\
\hline Riblet B & 0.5 & 30 & 1.27 \\
\hline
\end{tabular}

number range of $\mathrm{Re}_{d}=8.09 \cdot 10^{4}$ to $\mathrm{Re}_{d}=2.02 \cdot 10^{5}$. Values were calculated as:

$$
\operatorname{Re}_{d}=\frac{\rho \cdot u \cdot d}{\mu}
$$

with $\rho$ as density, $u$ as the free stream velocity, $d$ as the diameter of the circular body and $\mu$ as the dynamic viscosity.

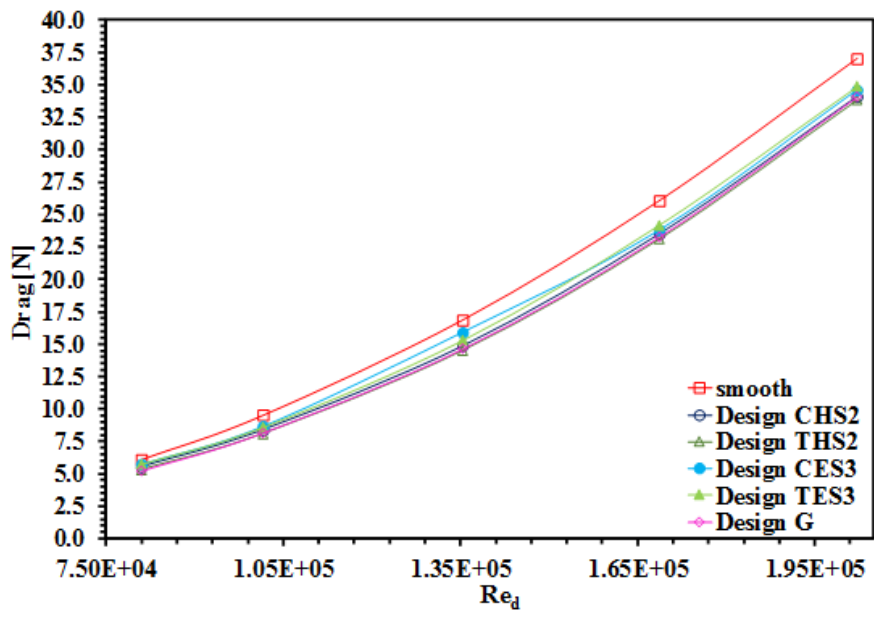

Fig. 5. Comparison of the two-dimensional non-smooth surfaces against the smooth surface. Drag as a function of the Reynolds number. C: circular;

T: triangular; G: golf ball; H: hollow; E: elevation; S: small.

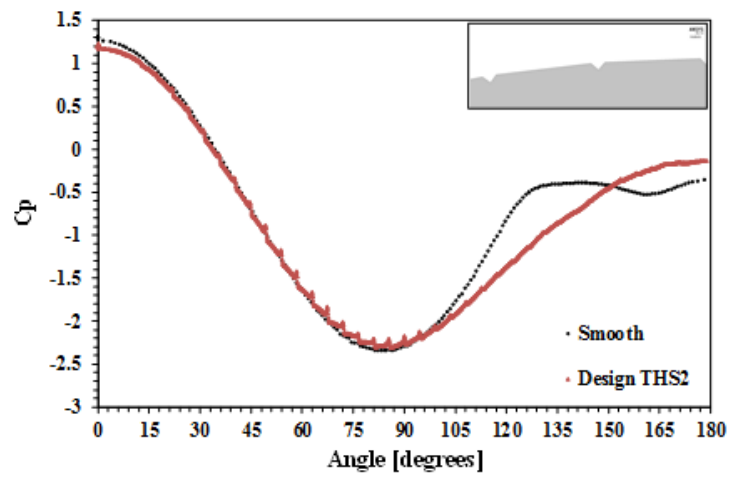

(a)

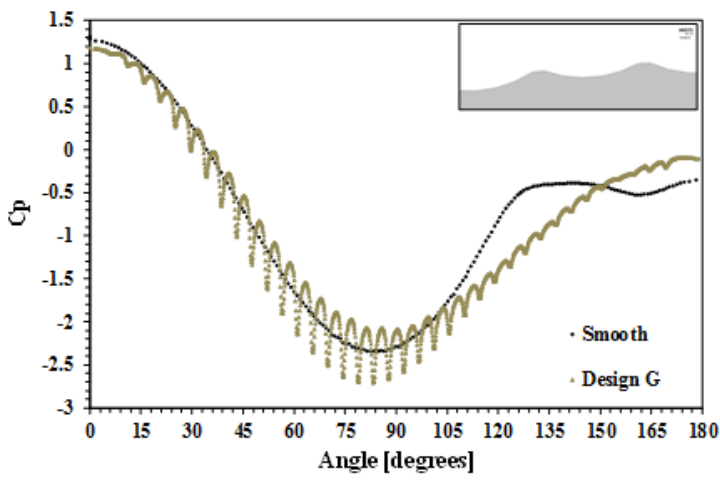

(b)

Fig. 5. Pressure coefficient around the circular body for $\operatorname{Re}_{d}=2.02 \cdot 10^{5}$ : (a) design THS2, (b) design G. T: triangular; G: golf ball; H: hollow; S: small. 


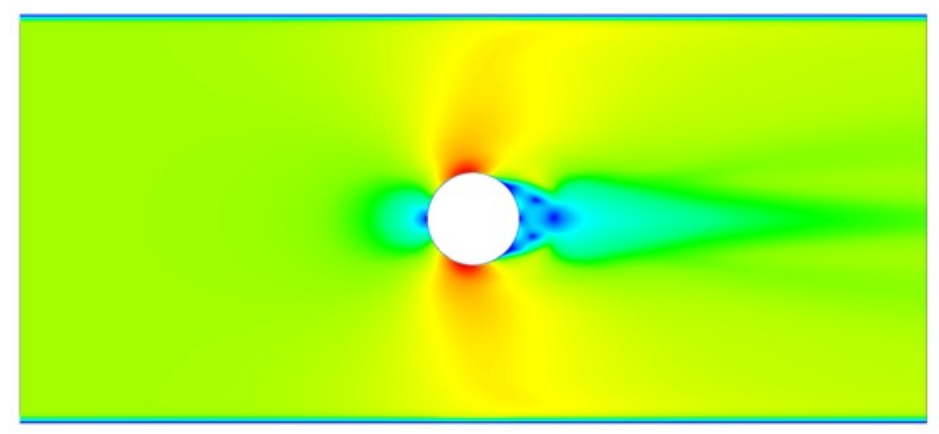

(a)

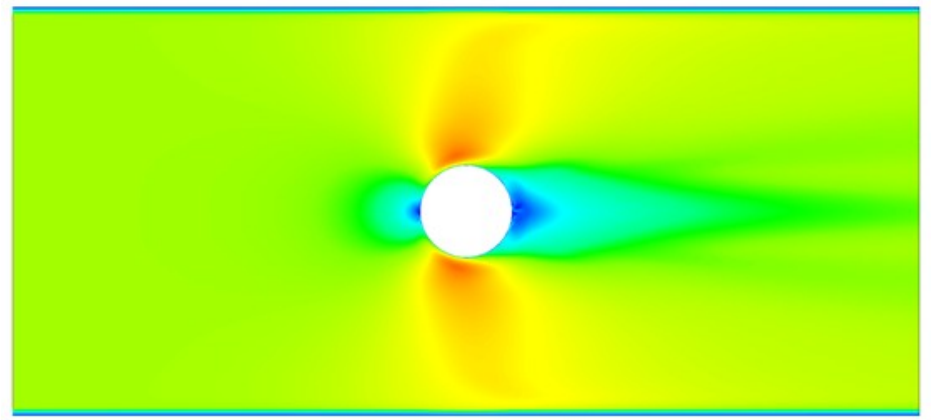

(c)

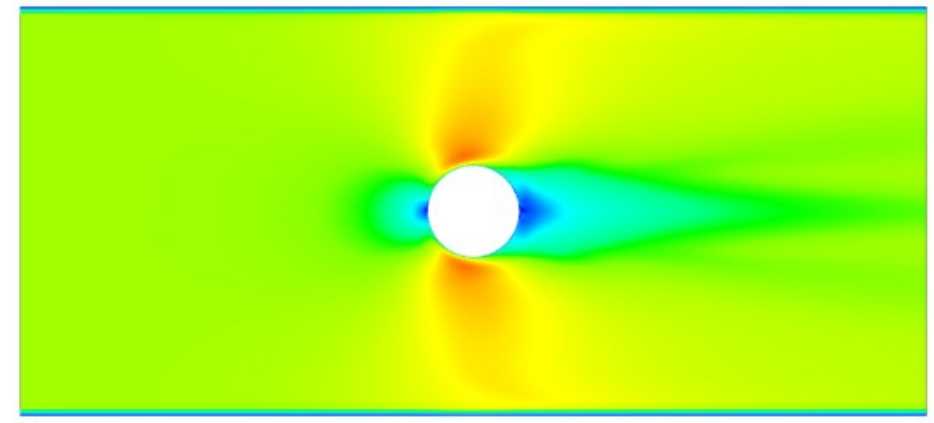

(e)

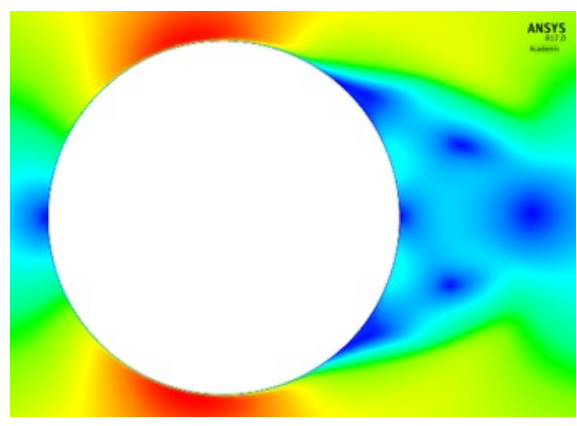

(b)

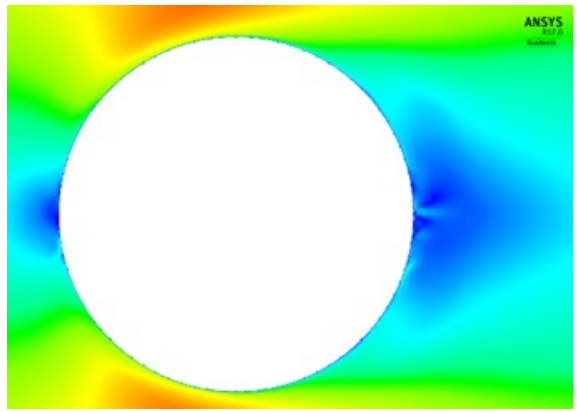

(d)

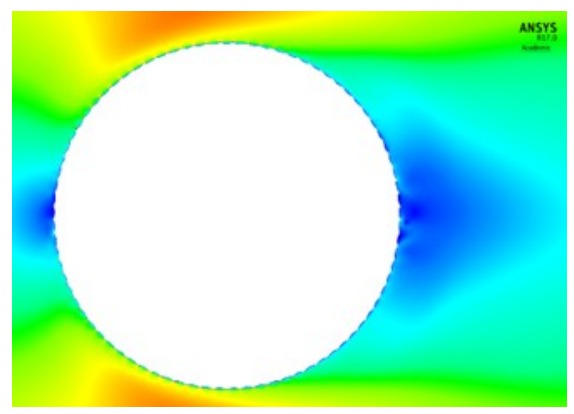

(f)

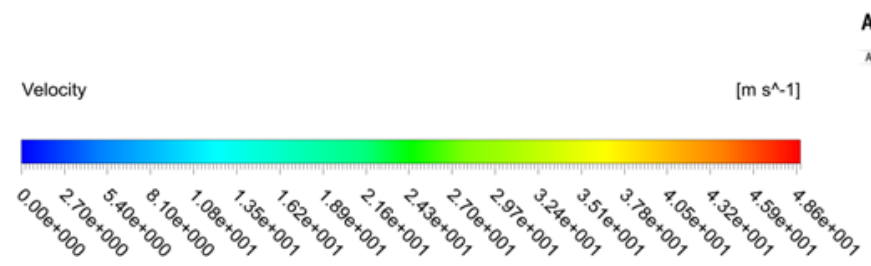

Fig. 7. Velocity contours for flow case $\mathrm{E}\left(\mathrm{Re}_{d}=2.02 \cdot 10^{5}\right)$ : (a) smooth geometry,

(b) close-up view of smooth geometry, (c) design THS2, (d) close-up view of design THS2, (e) design

$\mathrm{G}$, (f) close-up view of design $\mathrm{G},(\mathrm{g})$ velocity legend. T: triangular; G: golf ball; H: hollow; S: small.

\section{RESULTS AND DisCUSSION}

\section{A. Two-dimensional Analysis}

Mesh designs and solving models. The most congruent results in comparison with experimental data with an overall average deviation below $2.5 \%$ were obtained by using the standard k-epsilon model with scalable near-wall treatment and the hybrid mesh. Final meshes were generated not exceeding 200,000 cells.

The study of the flow around a circular cylinder in the Reynolds number range of $\mathrm{Re}_{d}=8.09 \cdot 10^{4}$ to $\mathrm{Re}_{d}=2.02 \cdot 10^{5}$ using a steady-state solution and the RANS approach support the ability to predict values of good agreement with experimental data. However, the pressure distribution was not accurately represented, in particular the prediction at the trailing part of the cylinder $\left(90^{\circ}<\theta<180^{\circ}\right)$. The predicted drag, on the other hand, agreed well with experimentally obtained values.

Analysis of non-smooth surfaces. Non-smooth surface designs were assessed about the ability to reduce drag for $\operatorname{Re}_{d}=2.02 \cdot 10^{5}$ in three main comparisons: the shape of the geometry, the size of the geometry and the number of uneven 


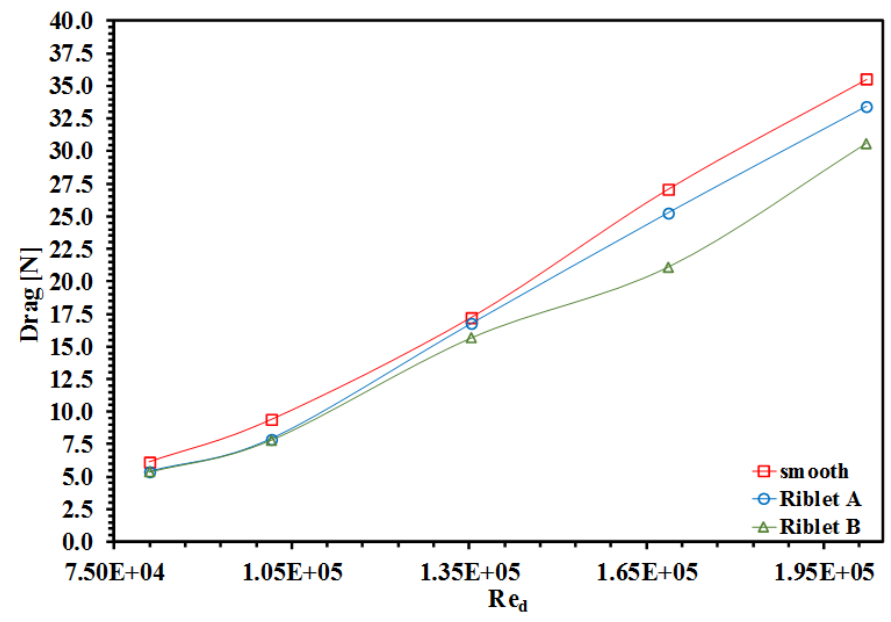

Fig. 8. Comparison of the three-dimensional riblet designs against the smooth surface. Drag as a function of the Reynolds number.

elements distributed. Circular geometries achieved in most of the simulations lower drag values than triangular geometries. Similar, hollow designs showed generally a higher reduction in drag than elevating designs. Structures with small elevating geometries show in comparison with designs of larger elevating geometries a higher drag reduction. On the other hand, the performance of small and large hollow structures showed a dependency of the space between the uneven elements. Moreover, elevating designs achieved the lowest drag values with the highest number of uneven elements while hollow designs performed superior with the lowest number of uneven elements.

Drag values of non-smooth surfaces are compared against values of the smooth surface in Fig. 5. The reduction in drag as a consequence of non-smooth structures which trigger turbulence and therefore shift the drag crisis, i.e. the minimum coefficient of drag to a lower Reynolds number range was observed. It was observed that crosswise to the free stream aligned riblets and golf ball similar structures were able of reducing the average drag force up to $12.45 \%$ within the Reynolds number range defined. Values of drag reduction were calculated as follow:

$$
\text { reduction }=\frac{F_{s}-F_{n}}{F_{s}} \cdot 100 \%
$$

where $F_{s}$ is drag for the smooth surface and $F_{n}$ is drag for the non-smooth surface. Positive values indicate a reduction in drag while negative values indicate an increase in drag force for uneven structures.

Fig. 6 shows the pressure coefficient of non-smooth surfaces in comparison with a smooth surface. The shift of the minimal turning point to higher degrees and the displacement of the minimum pressure region to higher angles in the cases of uneven structures indicates a later separation of the boundary layer. Moreover, the lower magnitude of the minimum pressure value for the prepared uneven designs reveals a smaller separated region behind the circular body.
Fig. 7 illustrates the velocity magnitude of the flow around the circular body for $\operatorname{Re}_{d}=2.02 \cdot 10^{5}$. Obviously, the flow at some distance around the circular body interferes with the wall boundaries. The smaller wake region with a higher velocity magnitude confirms the lower drag value and the transition to a turbulent boundary layer. Furthermore, it was observed that the flow on the surface around the cylinder separates at the leading edge of an indentation and is drawn down into the hollow shape before it reattaches at the trailing edge of the indentation. Therefore, the flow between the indentations along the circular body accelerates and triggers the transition from laminar to turbulent. As a consequence, the separation of the boundary layer delays and causes a reduction in drag force. In the case of elevations, the reduction of drag force was observed to be similar, with the difference being that the acceleration of the flow occurs as a result of the elevations directly at the geometry rather than between the geometries. This phenomenon of flow separation was also observed by Bogdanovic-Javonovic et al. [14] in the specific case of a three-dimensional golf ball.

\section{B. Three-dimensional Analysis}

The values of the three-dimensional simulation show very good agreement (average deviation below 0.5\%) in the comparison with the results experimentally obtained. Design Riblet A and Riblet B perform at low Reynolds numbers almost equal while in the range of higher Reynolds numbers, the design Riblet $\mathrm{B}$ outperforms design Riblet A. The design Riblet A which was dimensioned according to the relation of $h=0.5 s$ showed an average drag reduction of $8.41 \%$ while design Riblet B with a relation of $h \approx 0.4 s$ achieved an even higher average drag reduction of $14.7 \%$.

\section{CONCLUSION}

The key results of this investigation are summarised as follows.

- The crosswise aligned non-smooth surfaces in the configuration with semi-circular indentations generally achieved a higher drag reduction than triangular and elevating structures. However, a non-smooth surface with small triangular indentations was determined as optimized structure for the Reynolds number defined.

- It was observed that the velocity is reduced in the nearwall region of the non-smooth surface and that small vortices were generated within the structure cavities.

- Non-Smooth surface structures trigger turbulence and delay the boundary layer separation. As a consequence, a reduction in drag merges.

- Two-dimensional non-smooth structures designed achieved a drag reduction up to an average reduction of $12.45 \%$ while the from shark skin adapted threedimensional riblet structures achieved a drag reduction up to $14.7 \%$.

- The results presented corroborate the beneficial contribution of non-smooth surfaces to the aerodynamic behaviour of flow around a circular 
cylinder. Geometries and structures determined show specific improvements in the drag reduction and therefore provide the opportunity of designing structures with the potential to lower the air resistance.

\section{REFERENCES}

[1] Z. Han, Z. Mu, W. Yin, W. Li, S. Niu, J. Zhang, and L. Ren, "Biomimetic multifunctional surfaces inspired from animals," Advances in Colloid and Interface Science, vol. 234, pp. 27-50, 2016.

[2] A. Malshe, K. Rajurkar, A. Samant, and H.N. Hansen, "Bio-inspired functional surfaces for advanced applications," CIRP Annals Manufacturing Technology, vol. 62, no. 2, pp. 607-628, 2013.

[3] D.-Y. Zhao, Z.-P. Huang, M.-J. Wang, T. Wang, and Y. Jin, "Vacuum casting replication of micro-riblets on shark skin for drag-reducing applications," Journal of Materials Processing Technology, vol. 212, pp. 198-202, 2012.

[4] H. Chen, X. Zhang, L. Ma, D. Che, D. Zhang, and T.S. Sudarshan, "Investigation on large-area fabrication of vivid shark skin with superior surface functions," Applied Surface Science, vol. 316, pp. 124-131, 2014.

[5] Z. Dou, J. Wang, and D. Chen, "Bionic research on fish scales for drag reduction," Journal of Bionic Engineering, vol. 9, pp. 457-464, 2012.

[6] D.W. Bechert, and W. Hage, "Drag reduction with riblets in nature and engineering," in Flow Phenomena in Nature, vol. 1, A Challenge to Engineering Design, R. Liebe, Ed. Southampton: Wit Press, 2007, pp. 457-504.

[7] D.W. Bechert, M. Bruse, W. Hage, and J.G.T. Van der Hoeven, "Experiments on drag-reducing surfaces and their optimization with an adjustable geometry," Journal of Fluid Mechanics, vol. 338, pp. 59-87, 1997.

[8] O.A. El-Samni, H.H. Chun, and H.S. Yoon, "Drag reduction of turbulent flow over thin rectangular riblets," International Journal of Engineering Science, vol. 45, pp. 436-454, 2007.
[9] C. Xu, J. Wang, S. Luang, B. Qu, and L. Jiang, "Analysis of drag reduction mechanism of the bionic microscopic riblets surface," in Proc. 3rd Int. Conf. on Biomedical Engineering and Informatics, Yantai, China, 16-18 Oct. 2010, pp. 2394-2398.

[10] S. Martin, and B. Bhushan, "Modeling and optimization of sharkinspired riblet geometries for low drag applications," Journal of Colloid and Interface Science, vol. 474, pp. 206-215, 2016.

[11] L.-M. Tian, L.-Q. Ren, Q.-P. Liu, Z.-W. Han, and X. Jiang, "The mechanism of drag reduction around bodies of revolution using bionic non-smooth surfaces," Journal of Bionic Engineering, vol. 4, pp. 109116, 2007.

[12] B. Dean, and B. Bhushan, "The effect of riblets in rectangular duct flow," Applied Surface Science, vol. 258, pp. 3936-3947, 2012.

[13] G.D. Bixler, and B. Bhushan, "Shark skin inspired low-drag microstructured surfaces in closed channel flow," Journal of Colloid and Interface Science, vol. 393, pp. 384-396, 2013.

[14] J.M. Bogdanovic-Jovanovic, "Experimental and numerical investigation of flow around a sphere with dimples for various flow regimes," Thermal Science, vol. 16, no. 4, pp. 1013-1023, 2012.

[15] H. Chowdhury, B. Loganathan, Y. Wang, I. Mustary, and F. Alam, "A study of dimple characteristics on golf ball drag," Procedia Engineering, vol. 147, pp. 87-91, 2016.

[16] K. Zhang, H. Katsuchi, D. Zhou, H. Yamada, and Z. Han, "Numerical study on the effect of shape modification to the flow around circular cylinder," Journal of Wind Engineering and Industrial Aerodynamics, vol. 152, pp. 23-40, 2016.

[17] J. Cui, and Y. Fu, "A numerical study on pressure drop in microchannel flow with different bionic micro-grooved surfaces," Journal of Bionic Engineering, vol. 9, pp. 99-109, 2012.

[18] Y. Zhiyin, "Large-eddy simulation: Past, present and the future," Chinese Journal of Aeronautics, vol. 28, no. 1, pp. 11-24, 2015. 